\title{
Identification of Potential Biomarkers and Immune Infiltration Characteristics in Idiopathic Pulmonary Arterial Hypertension Using Bioinformatics Analysis
}

\author{
Haowei Zeng, Xiaoqin Liu and Yushun Zhang* \\ Department of Structural Heart Disease, The First Affiliated Hospital of Xi'an Jiaotong University, Xi'an, China
}

\section{OPEN ACCESS}

Edited by:

Shizuka Uchida,

Aalborg University

Copenhagen, Denmark

Reviewed by:

Y.-h. Taguchi,

Chuo University, Japan

Peixin Dong,

Hokkaido University, Japan

Kenji Satou,

Kanazawa University, Japan

*Correspondence:

Yushun Zhang

zys2889@sina.com

Specialty section:

This article was submitted to

Atherosclerosis and Vascular

Medicine,

a section of the journal

Frontiers in Cardiovascular Medicine

Received: 01 November 2020 Accepted: 04 January 2021

Published: 01 February 2021

Citation:

Zeng H, Liu X and Zhang Y (2021) Identification of Potential Biomarkers and Immune Infiltration Characteristics

in Idiopathic Pulmonary Arterial

Hypertension Using Bioinformatics

Analysis.

Front. Cardiovasc. Med. 8:624714.

doi: 10.3389/fcvm.2021.624714
Objectives: Idiopathic pulmonary arterial hypertension (IPAH) is a rare but severe lung disorder, which may lead to heart failure and early mortality. However, little is known about the etiology of IPAH. Thus, the present study aimed to establish the differentially expressed genes (DEGs) between IPAH and normal tissues, which may serve as potential prognostic markers in IPAH. Furthermore, we utilized a versatile computational method, CIBERSORT to identify immune cell infiltration characteristics in IPAH.

Materials and Methods: The GSE117261 and GSE48149 datasets were obtained from the Gene Expression Omnibus database. The GSE117261 dataset was adopted to screen DEGs between IPAH and the control groups with the criterion of |log2 fold change| $\geq 1$, adjusted $P<0.05$, and to further explore their potential biological functions via Gene Ontology analysis, Kyoto Encyclopedia of Genes and Genomes Pathway analysis, and Gene Set Enrichment Analysis. Moreover, the support vector machine (SVM)-recursive feature elimination and the least absolute shrinkage and selection operator regression model were performed jointly to identify the best potential biomarkers. Then we built a regression model based on these selected variables. The GSE48149 dataset was used as a validation cohort to appraise the diagnostic efficacy of the SVM classifier by receiver operating characteristic $(\mathrm{ROC})$ analysis. Finally, immune infiltration was explored by CIBERSORT in IPAH. We further analyzed the correlation between potential biomarkers and immune cells.

Results: In total, 75 DEGs were identified; 40 were downregulated, and 35 genes were upregulated. Functional enrichment analysis found a significantly enrichment in heme binding, inflammation, chemokines, cytokine activity, and abnormal glycometabolism. HBB, RNASE2, S100A9, and IL1R2 were identified as the best potential biomarkers with an area under the ROC curve (AUC) of 1 $(95 \% \mathrm{Cl}=0.937-1.000$, specificity $=100 \%$, sensitivity $=100 \%)$ in the discovery cohort and $1(95 \% \mathrm{Cl}=0.805-1.000$, specificity $=100 \%$, sensitivity $=100 \%)$ in the validation cohort. Moreover, immune infiltration analysis by CIBERSORT showed a higher level of CD8+ T cells, resting memory CD4+ T cells, gamma delta T cells, M1 macrophages, resting mast cells, as well as a lower level of nailve CD4+ $T$ cells, monocytes, 
MO macrophages, activated mast cells, and neutrophils in IPAH compared with the control group. In addition, HBB, RNASE2, S100A9, and IL1R2 were correlated with immune cells.

Conclusion: $H B B, R N A S E 2, S 100 A 9$, and IL1R2 were identified as potential biomarkers to discriminate IPAH from the control. There was an obvious difference in immune infiltration between patient with IPAH and normal groups.

Keywords: idiopathic pulmonary arterial hypertension, immune infiltration, inflammation, biomarkers, bioinformatics

\section{INTRODUCTION}

Pulmonary arterial hypertension (PAH) is a progressive cardiopulmonary disease in which increased pulmonary arterial pressure leads to right ventricle failure and premature death. $\mathrm{PAH}$ is defined as mean pulmonary artery pressure $(\mathrm{mPAH}) \geq$ $25 \mathrm{mmHg}$, pulmonary artery wedge pressure $\leq 15 \mathrm{mmHg}$ and pulmonary vascular resistance (PVR) $>3$ Wood units. There are several categories of reasons for patients to develop PAH, but in the absence of any known triggering factor, they are labeled as idiopathic PAH (IPAH) (1). It is progressive and affects the precapillary pulmonary vasculature. It is rare but fatal with a high mortality rate, and its prevalence accounts vary among the medical literature (2). IPAH may lead to increased PVR, progressive right ventricular dysfunction, right heart failure, and early mortality. The symptoms of IPAH are non-specific, including fatigue, weakness, shortness of breath, angina, and syncope, usually induced by exertion $(1,3,4)$. Although the cause of IPAH is unclear, some cases are familial and caused by gene defects, or mutations, such as BMPR2 (5), SMAD8 (6), ALK1 (7), and $B M P R 1 B(8)$ and so on. Additionally, environmental factors, such as acute hypoxia may be involved in IPAH (9). The diagnosis of IPAH requires the identification of symptoms and a physical examination and a comprehensive set of investigations to guarantee that hemodynamic criteria are met and to describe the etiology as well as the functional and hemodynamic severity of the condition. Despite extensive researches and improved diagnosis as well as a broad range of increasing biomarkers, there is currently no specific marker for IPAH. This disorder is still associated with a poor prognosis, especially when complicated with arrhythmia, left ventricular dysfunction, or heart failure. Therefore, prompt and specific diagnoses are needed to reduce mortality rates.

Although the specific pathophysiology remains poorly understood, increasing evidence suggests a crucial role of inflammation in IPAH. Patients with IPAH showed an accumulation of immune cells around the vessel, including dendritic cells (DCs), B and T lymphocytes, macrophages, and mast cells. Additionally, circulating levels of several chemokines and cytokines are also increased (10-15).

Recently, gene expression microarray studies have been extensively applied to find potential biomarkers and their roles in complicated diseases to further explore the pathogenesis and develop potential treatments (16). In this study, the GSE117261 dataset was adopted to screen differentially expressed genes (DEGs) between IPAH and a control group, and to further explore their potential biological functions via enrichment analysis. We identified the best feature genes that could distinguish IPAH from controls. Finally, immune infiltration was investigated in IPAH by CIBERSORT (17).

\section{MATERIALS AND METHODS}

\section{IPAH Datasets}

The GSE117261 and GSE48149 datasets were downloaded from the NCBI Gene Expression Omnibus (GEO) database (https://www.ncbi.nlm.nih.gov/geo/). GSE117261 was based on the GPL6224 Affymetrix Human Gene 1.0 ST Array. It consists of transcript profiles of lung tissues from 25 normal individuals and 32 patients with IPAH, which served as a discovery cohort. Its expression value was normalized using the "limma" package (18) in R software (version 4.0.2; https://www.r-project.org/) to make sure the expression levels had a similar distribution among a set of arrays. GSE48149 was based on GPL16221 Illumina HumanRe-8 v3.0 expression beadchip. The lung tissues of nine normal individuals and eight patients with IPAH were obtained during lung surgery, which served as a validation cohort. Its expression level was normalized and $\log 2$ transformed by R software.

\section{Identification of DEGs}

To establish the DEGs between the IPAH and the control group, the "limma" package (18) was adopted in R software. Moreover, a volcano plot was generated to assess the DEGs, |log2 fold change (FC) $\mid \geq 1$, adjusted $P$ value $<0.05$ was set as a cutoff for this selection.

\section{Functional Enrichment of DEGs}

The Gene Ontology (GO) analysis, Kyoto Encyclopedia of Genes and Genomes (KEGG) pathway analysis, and Gene Set Enrichment Analysis (GSEA) were carried out to annotate and analyze the biological functions of the DEGs. The GO project provides a set of hierarchical controlled vocabulary divided into three categories: cellular component, biological process and molecular function. The KEGG pathway is a group of pathway maps showing the knowledge of the molecular interaction, reaction and relation networks. GSEA is a computational approach that decides whether an a priori 
A

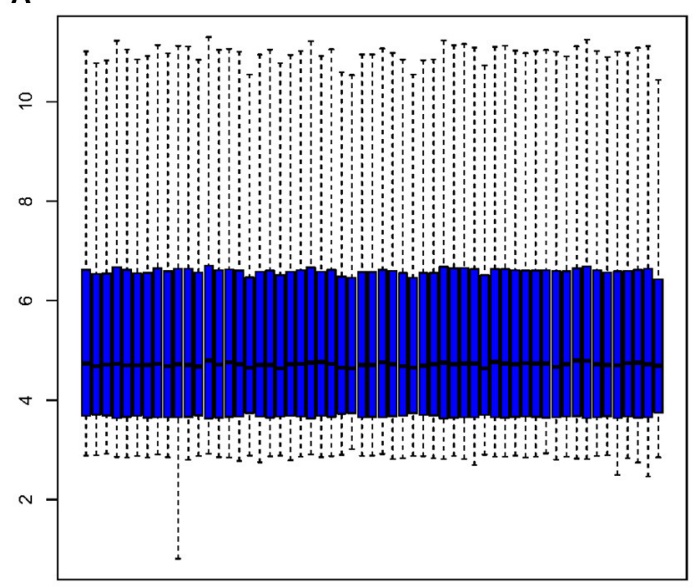

C

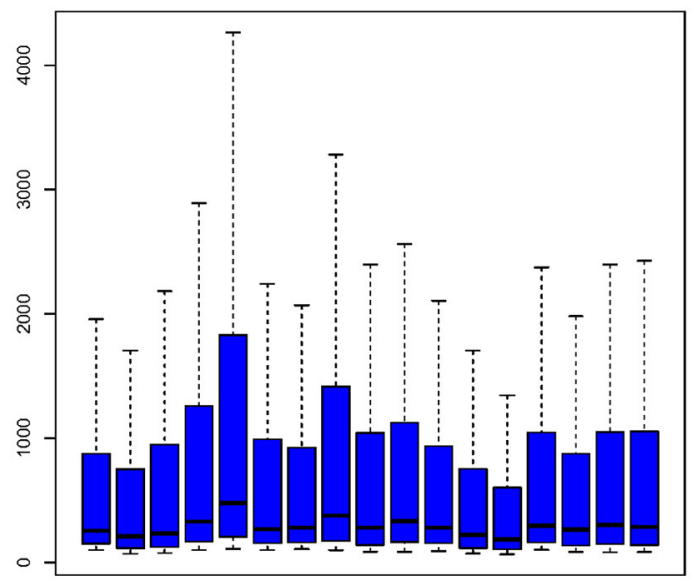

B

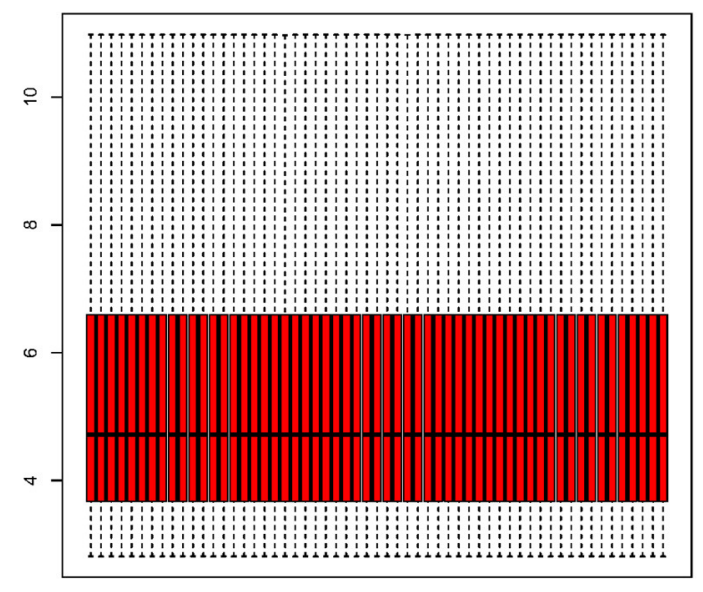

D

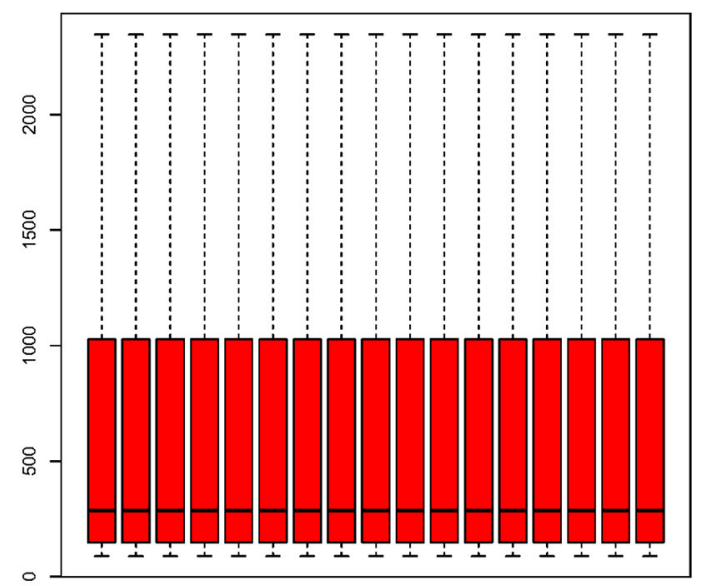

FIGURE 1 | The box diagram of expression profile before and after normalization. GSE117261 expression profile before (A) and after (B) normalization; GSE48149 expression profile before (C) and after (D) normalization.

defined set of genes shows statistically significant differences between two groups. R software and the "clusterProfiler" package (19) were used to conduct GO analysis and KEGG analysis. In this analysis symbol codes were converted to Entrez ID using Human genome annotation package "org.Hs.eg.db." We used the "ggplot2" (20), "pathview" (21) and "gplots" packages of $\mathrm{R}$ software to visualize the plots. GSEA software (version 4.1.0, http://www.gsea-msigdb.org/ gsea/index.jsp) was used to carry out GSEA analysis (22). The "c2.cp.kegg.v7.0.symbols.gmt" was chosen as the reference gene set. Gene set permutations were performed 1,000 times for each analysis. Adjusted $P$-value $<0.05$ and $Q$ value $<0.05$ were considered significant for GO and KEGG analysis. The cutoff point of significance was |normalized enrichment score $(\mathrm{NES}) \mid>1, P$-value $<0.05$, false discovery rate $(\mathrm{FDR}) \mathrm{Q}$ value $<$ 0.25 for GSEA.

\section{Feature Gene Identification and Support Vector Machine Classifier Construction}

Support vector machine (SVM) is a supervised machine learning algorithm that can be used for regression or classification, which is derived on the basis of a training cohort that detects patterns in the data and associates them with labels (23). The SVM -recursive feature elimination (RFE) is a machine learning algorithm requires training multiple classifiers on subsets of features of decreasing size to search for the best variable (24). The least absolute shrinkage and selection operator (LASSO) regression model was further conducted to select optimal variables with the use of penalty coefficient. The LASSO logistic regression method and the SVM-RFE were used to select the most significant feature genes and build a regression model using the selected variables $(25,26)$. The performance of the SVM classifier in distinguishing IPAH from control groups was assessed by the area under the 


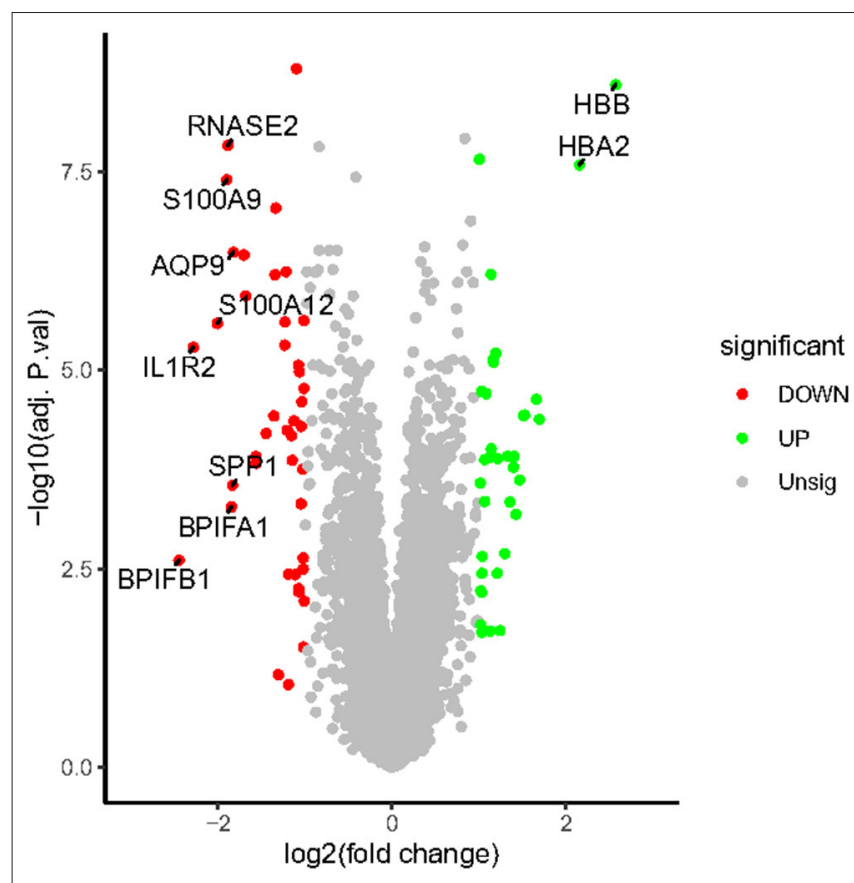

FIGURE 2 | The volcano map of DEGs. The plot compared the DEGs between IPAH patients and controls from the GSE117261 dataset. The red dots represent the down-regulated DEGs; the green dots represent up-regulated DEGs; gray dots indicate the remaining genes that were not significantly changed. Genes signed in the plot are the top ten genes with the most significant logFC.

receiver operating characteristic (ROC) curve (AUC) in the discovery and validation cohorts. The "glmnet" package (27) was adopted to analyze LASSO regression. The "e1071" package (28) was used to perform the SVM algorithm. The "pheatmap" package (29) was used to construct a heat map of genes for all samples in both the discovery and validation cohorts.

\section{Immune Infiltration Analysis}

CIBERSORT, a method of analyzing the different immune cell types of tissues using their gene expression profiles (30), was adopted to analyze the normalized GSE117261 expression data. Next, a matrix of 22 kinds of immune cells was obtained. CIBERSORT $P<0.05$ was used to filter the samples. And the percentage of each immune cell type in the samples was calculated and displayed in a bar plot. A heat map of the 22 immune cells was constructed using the "pheatmap" package (29). The "vioplot" package (31) was used to compare the levels of 22 immune cells between the two groups. A correlation heatmap, which revealed the correlation of 22 kinds of immune cells, was made by the "corrplot" package (32) of R software. The "ggplot2" package (20) was adopted to visualize the plots.

\section{Correlation Analysis Between Immune Cells and Feature Genes}

Spearman's rank correlation analysis was used to analyze the relationship between immune cells and feature genes in $R$ software. The "ggplot2" package (20) was adopted to visualize the plot, $P$-value $<0.05$ was considered statistically significant.

\section{Statistical Analysis}

R software (version 4.0.2; https://www.r-project.org/) was used for all statistical analysis. Continuous variables are expressed as mean $\pm S D$, and differences between two groups were compared using Student's $t$-test for normally distributed variables and Mann-Whitney U test for abnormally distributed variables. The sensitivity and specificity of feature genes to distinguish IPAH from controls were assessed using a ROC curve. Adjusted $P$-value $<0.05$ was considered statistically significant.

\section{RESULTS}

\section{Data Preprocessing and Identification of DEGs}

All expression values of the GSE117261 and GSE48149 datasets were normalized. And the data before and after normalization are presented as box diagrams in Figure 1. In total, 75 DEGs were identified: 40 were downregulated and 35 genes were upregulated. The volcano plot showed (Figure 2) that BPIFB1, IL1R2, S100A12, S100A9, RNASE2, BPIFA1, SPP1, AQP9, HBA2, and $H B B$ were the top ten genes with the most significant $\log F C$, and were used to further identify potential biomarkers.

\section{Functional Enrichment Analysis of DEGs}

Go enrichment analysis demonstrated that DEGs were mainly associated with heme binding (GO:0020037), chemokine binding (GO:0019956), cytokine binding (GO:0019955), immune receptor activity (GO:0140375), cytokine receptor activity (GO:0004896), C-C chemokine binding (GO:0019957), $\mathrm{C}-\mathrm{C}$ chemokine receptor activity (GO:0016493), and chemokine receptor activity (GO:0004950) (Figure 3A). The KEGG enrichment analysis was primarily involved in fluid shear stress and atherosclerosis (hsa05418), cytokine-cytokine receptor interaction (hsa04060), viral protein interaction with cytokine and cytokine receptor (hsa04061) (Figure 3B). In the GSEA analysis, the citrate cycle (TCA cycle) (hsa00020, NSE $=-1.83$, normal $P$-value $=0.004$, FDR $=0.108)$ and starch and sucrose metabolism (hsa00500, NSE $=-1.66$, normal $P$-value $=0.004$, $\mathrm{FDR}=0.204$ ) were remarkably suppressed in patients with IPAH (Figures 3C,D). In summary, functional enrichment showed the DEGs mainly involved in heme binding, inflammation, chemokine, cytokine activity, and abnormal glycometabolism.

\section{Feature Genes Selection and SVM Classifier Construction}

To select the best biomarkers of IPAH, the top ten genes with the most significant $\log F C$ were further analyzed. The LASSO logistic regression method was used to identify five potential biomarkers (Figures 4A,B): HBB, RNASE2, S100A9, IL1R2, and BPIFB1. The SVM-RFE identified eight (Figure 4C): RNASE2, HBB, S100A9, HBA2, SPP1, AQP9, IL1R2, and $S 100 A 12$. Eventually, we got four diagnostic genes by the two algorithms overlapped (Figure 4D): HBB, RNASE2, S100A9, and IL1R2, of which $H B B$ was upregulated, whereas RNASE2, 
A

C

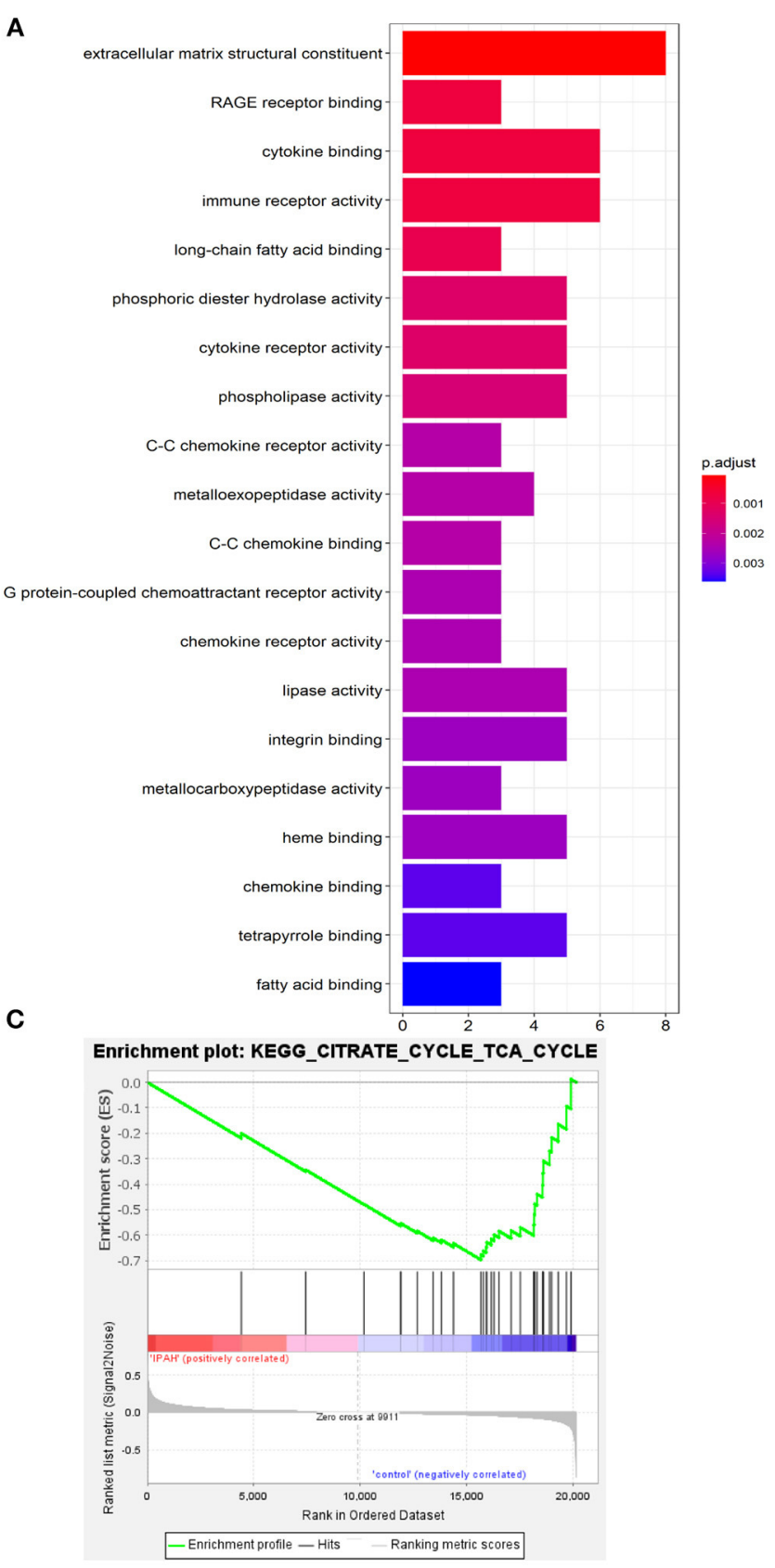

B

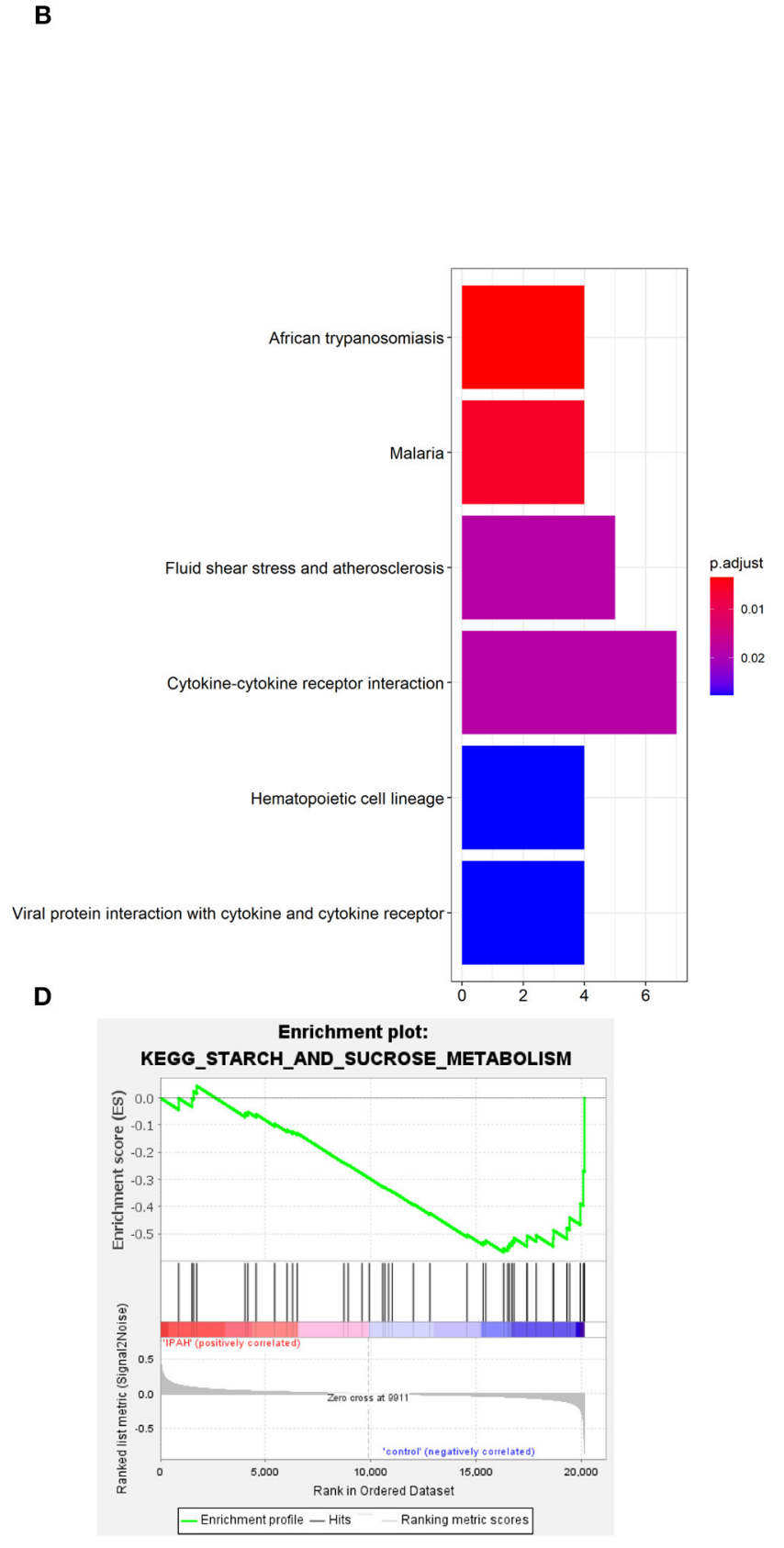

FIGURE 3 | The functional enrichment analysis of DEGs. (A) The GO enrichment; (B) The KEGG enrichment; (C,D) The GSEA enrichmen analysis.

S100A9, and IL1R2 were downregulated. Then, the ROC analysis was used to further appraise the predictive value of the four feature genes, respectively, and jointly in the diagnosis of IPAH in the two cohorts. The AUC was 0.954 $(95 \% \mathrm{CI}=0.863-0.992)$ for $\mathrm{HBB}, 0.961(95 \% \mathrm{CI}=0.874-0.995)$ for RNASE2, $0.931(95 \% \mathrm{CI}=0.832-0.981)$ for S100A9, 0.894 $(95 \% \mathrm{CI}=0.784-0.960)$ for $I L 1 R 2$, and 1 (95\%CI $=0.937-$ 1.000) when these four genes combined into one variable (Figures 5A-E) in GSE117261 dataset. And the AUC was 0.917
(95\%CI $=0.680-0.995)$ for $\mathrm{HBB}, 0.958(95 \% \mathrm{CI}=0.738-1.000)$ for RNASE2, 0.722 (95\%CI $=0.457-0.907)$ for S100A9, 0.708 (95\%CI $=0.443-0.898)$ for IL1R2, and 1 (95\%CI $=0.805-$ 1.000) when these four genes combined into one variable in GSE48149 dataset (Figures 5F-J). Hierarchical clustering analysis in both the discovery (Figure 6A) and validation cohorts (Figure 6B) suggested that the patients were clearly divided into two groups based on the expression levels of these four DEGs. Figure 6C showed the different expression 
A

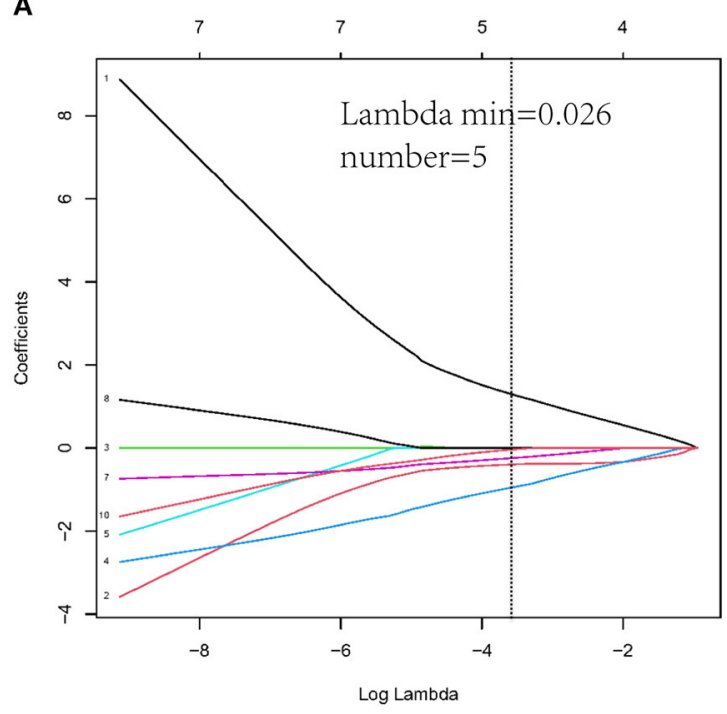

C

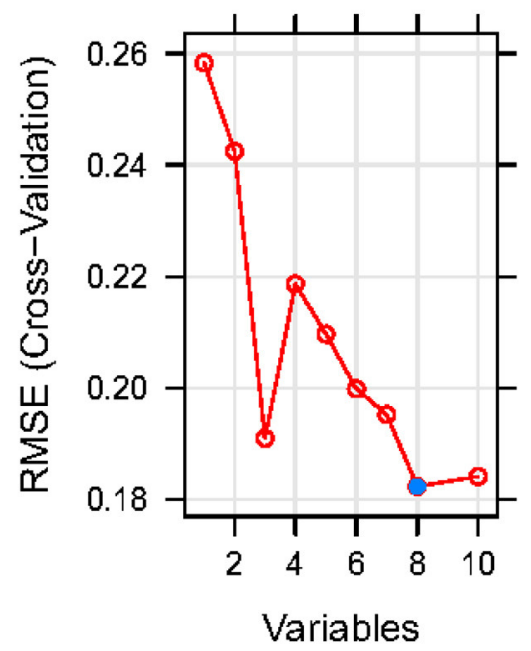

B
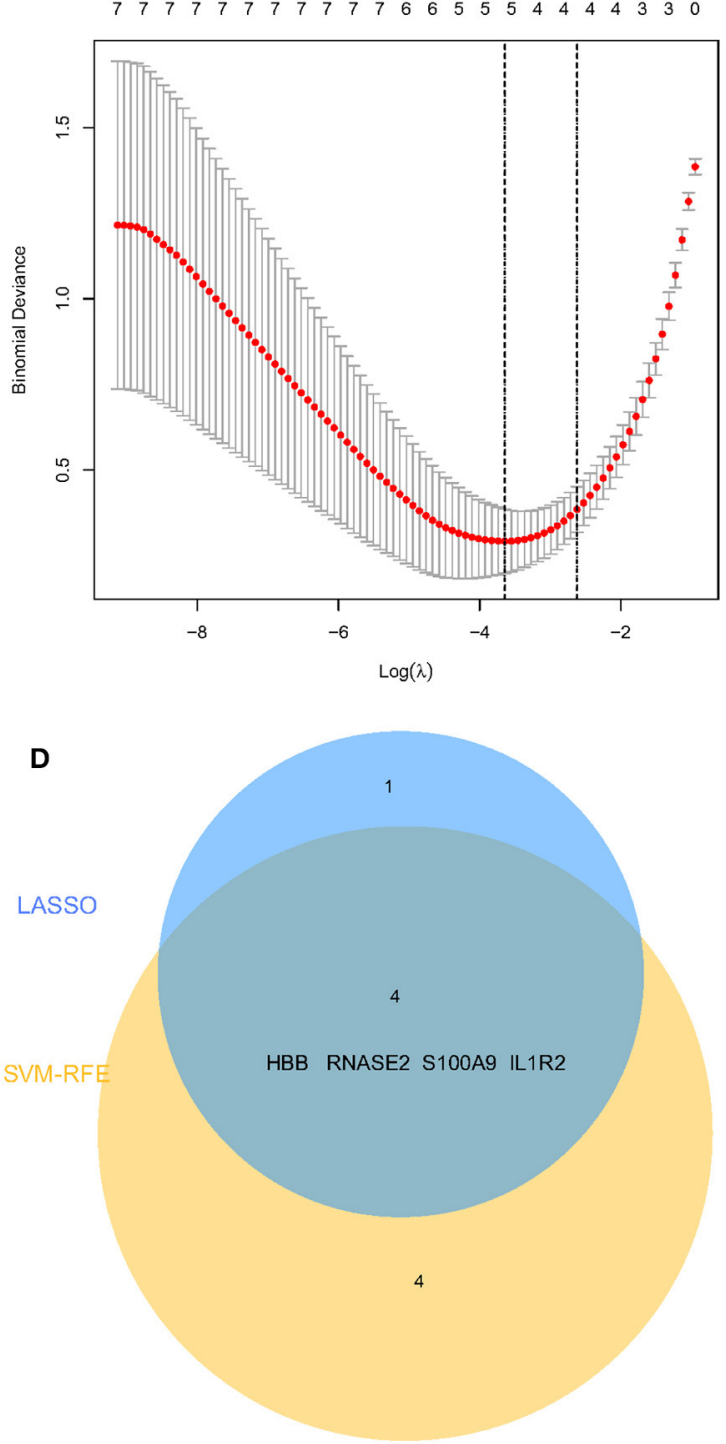

FIGURE 4 | Establishment of prognostic genes signature by LASSO regression analysis and SVM-RFE algorithm. (A) LASSO coefficient profiles of five genes. The dotted vertical line is the value selected using the 10-fold cross-validation in (B), for which the optimal lambda $\lambda$ ) resulted in five non-zero coefficients. (B) Identification of the optimal penalization coefficient $(\lambda)$ in the Lasso model used 10-fold cross-validation and the minimum absolute contraction criterion. (C) A plot of feature gene selection by SVM-RFE. The blue dot represents the best eight variables; (D) The Venn plot of overlapped feature genes between LASSO regression analysis and SVM-RFE algorithm.

level of these four genes between IPAH and the control in the validation cohort. The result suggested that these four genes have a good diagnostic value for discriminating IPAH from the control group.

\section{Immune Infiltration Analysis}

Functional enrichment analysis of DEGs showed an obvious enrichment in immune response. Hence, we further performed the CIBERSORT algorithm to predict the immune cells infiltration between patients with IPAH and the control group. The percentage of each of the 22 types of immune cells in each sample was shown in the bar plot and heat map (Figures $7 \mathrm{~A}, \mathbf{B})$. The vioplot of the immune cell infiltration difference demonstrated that IPAH patients had a higher level of $\mathrm{CD} 8+\mathrm{T}$ cells, resting memory $\mathrm{CD} 4+\mathrm{T}$ cells, gamma delta $\mathrm{T}$ cells, $\mathrm{M} 1$ macrophages, resting mast cells, and a lower level of naïve $\mathrm{CD} 4+\mathrm{T}$ cells, monocytes, M0 macrophages, activated mast cells, and neutrophils compared with the control group (Figure 7C). A correlation heatmap of immune cells revealed that gamma delta $\mathrm{T}$ cells were positively related with $\mathrm{M} 1$ macrophages $(r=0.69)$ and CD8+ $\mathrm{T}$ cells $(r=0.67)$, neutrophils were positively related with 


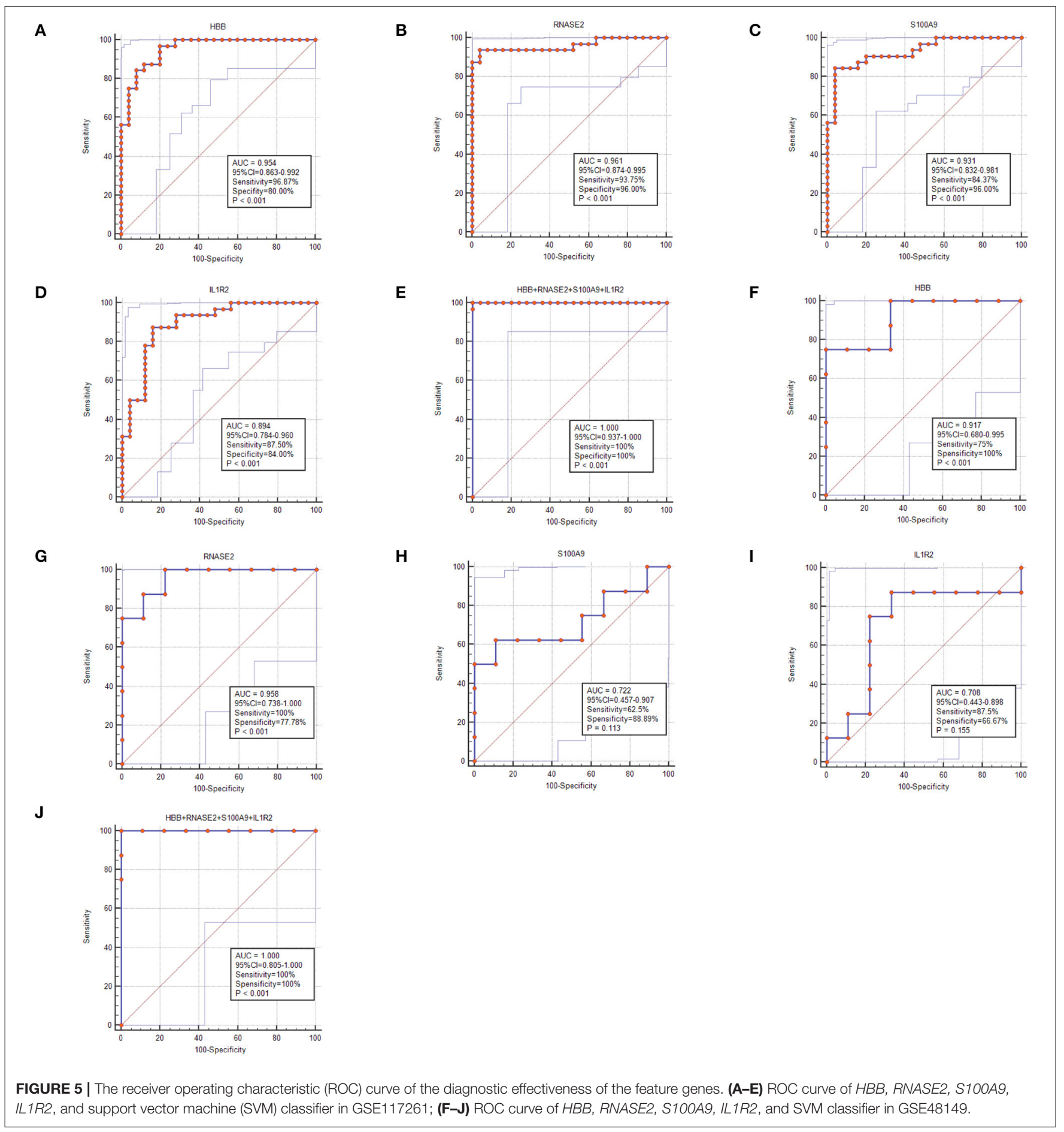

monocytes $(r=0.62)$, activated NK cells were positively related with follicular helper $\mathrm{T}$ cells $(r=0.58), \mathrm{M} 1$ macrophages were positively related with activated memory $\mathrm{CD} 4+\mathrm{T}$ cells $(r=0.47)$, whereas resting memory $\mathrm{CD} 4+\mathrm{T}$ cells were negatively related to M0 macrophages $(r=-0.5)$, resting mast cells were negatively related to neutrophils $(r=-0.49)$ (Figure 7D). To conclude, there is a significant difference in immune cell infiltration between patients with IPAH and controls.

\section{The Correlation Between HBB, RNASE2, S100A9, and IL1R2 and Immune Cells}

The correlation analysis revealed that $H B B$ (Figure 8A) had a positive correlation with memory resting CD4+ T cells $(r=0.36$, 


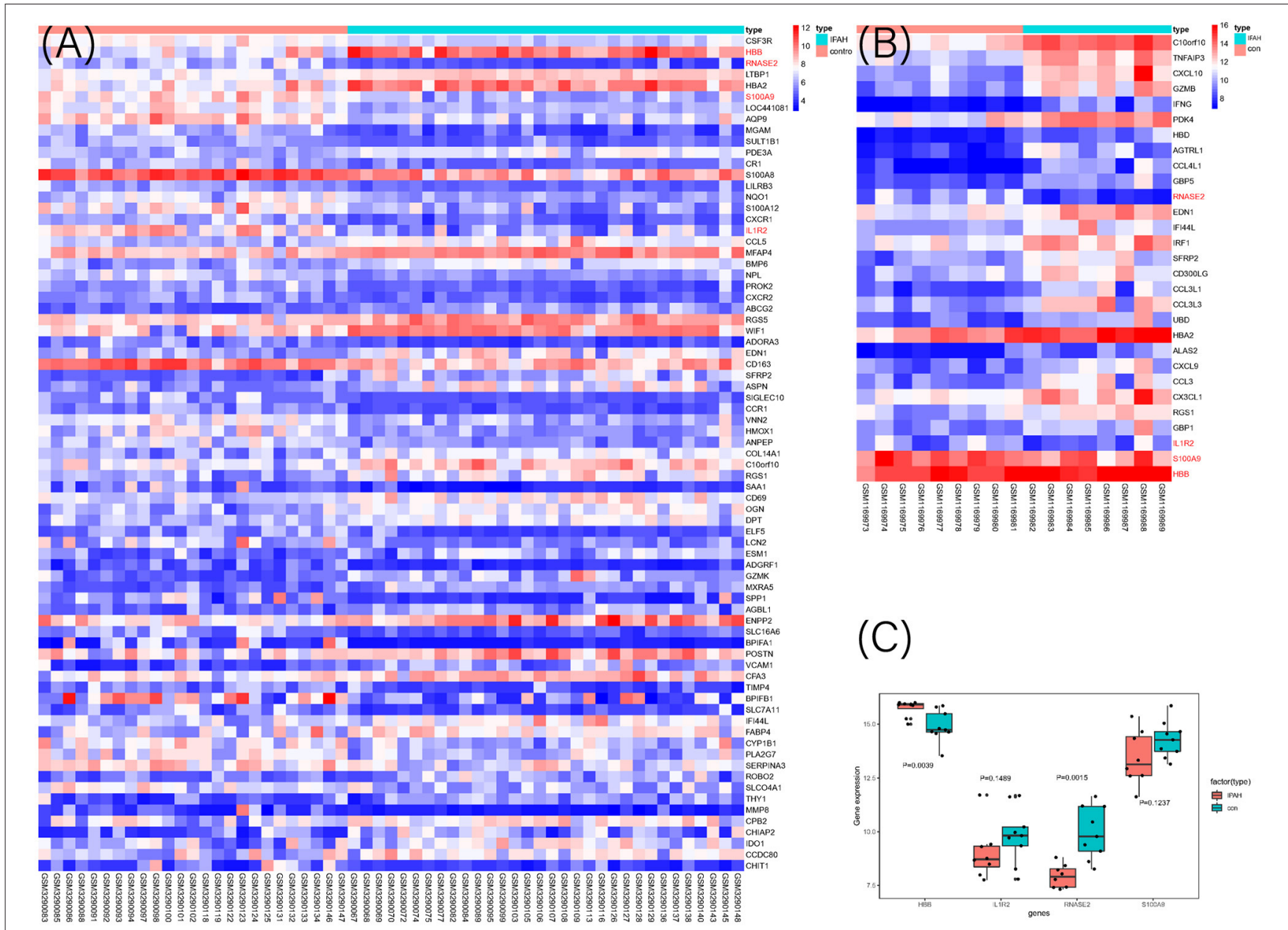

FIGURE 6 | The heat map of DEGs in discovery cohort (A) and validation cohort (B) between IPAH and the control group. The selected four genes were marked as red color; (C) The expression value of the identified four genes in the validation cohort.

$P=0.006)$, and negative correlation with neutrophils $(r=-0.35$, $P=0.007)$ and monocytes $(r=-0.38, P=0.003)$. RNASE2 (Figure 8B) had a positive correlation with naïve $\mathrm{CD} 4+\mathrm{T}$ cells $(r=-0.37, P=0.005)$, neutrophils $(r=0.65, P<$ $0.001)$, monocytes $(r=0.75, P<0.001)$, and resting NK cells $(r=0.33, P=0.01)$, and negative correlation with resting mast cells $(r=-0.33, P=0.01), \mathrm{CD} 8+\mathrm{T} \operatorname{cells}(r=-0.32$, $P=0.01)$, and resting memory CD4+ $\mathrm{T}$ cells $(r=-0.32$, $P=0.02)$. S100A9 (Figure 8C) had a positive correlation with monocytes $(r=0.76, P<0.001)$, neutrophils $(r=0.82$, $P<0.001)$, resting NK cells $(r=0.39, P=0.003)$, naïve CD4+ T cells $(r=0.40, P=0.002)$, and negative correlation with resting mast cells $(r=-0.48, P<0.001), \mathrm{CD} 8+\mathrm{T}$ cells $(r=-0.40, P=0.002)$, resting memory CD4+ T cells $(r=-0.34, P=0.009)$, and plasma cells $(r=-0.33, P=0.01)$. IL1R2 (Figure 8D) had a positive correlation with monocytes $(r=0.43, P<0.001)$, neutrophils $(r=0.66, P<0.001)$, and negative correlation with resting mast cells $(r=-0.32$, $P=0.02)$, follicular helper $\mathrm{T}$ cells $(r=-0.31, P=0.02)$, and CD8+ T cells $(r=-0.30, P=0.02)$. In conclusion,
$H B B, R N A S E 2, S 100 A 9$, and IL1R2 were all correlated with immune cells.

\section{DISCUSSION}

This study demonstrated a striking difference in the genes between IPAH and the control group. In total, 75 DEGs were identified: 35 genes were upregulated, and 40 were downregulated. We then used the LASSO regression model and SVM-RFE algorithm to select the best potential biomarkers with excellent diagnostic value: HBB, RNASE2, S100A9, and IL1R2. Moreover, the functional enrichment analysis revealed the DEGs were significantly involved in heme binding, inflammation, chemokines, cytokine activity, and abnormal glycometabolism. And we found an obvious difference in immune infiltration between patients with IPAH and controls by CIBERSORT algorithm. Furthermore, we found that these four genes were all correlated with immune cells. Previous studies have investigated the role of inflammation, chemokine, and cytokines in $\mathrm{PAH}$, but 

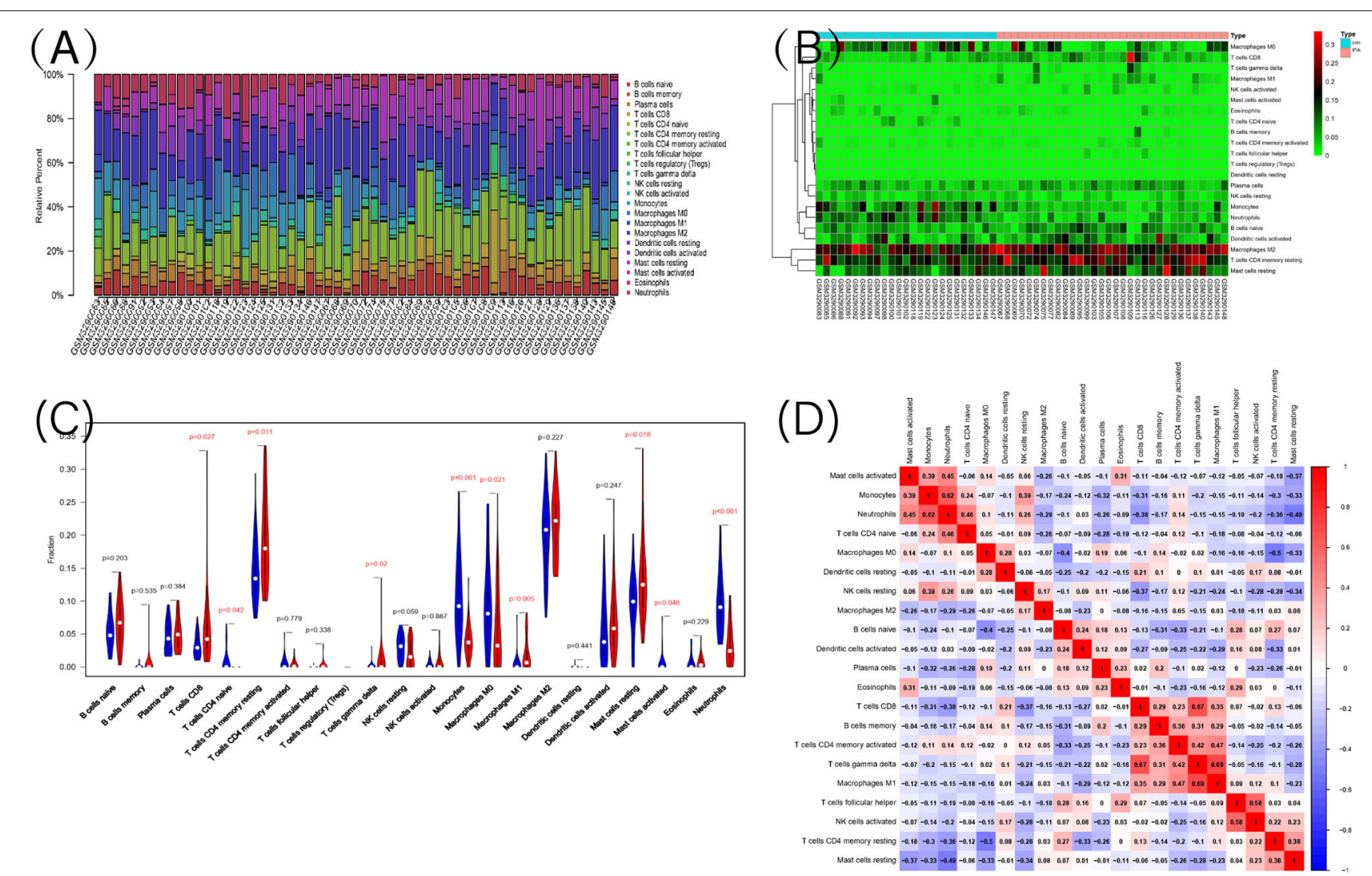

FIGURE 7 | The landscape of immune infiltration between IPAH and normal controls. (A) The relative percentage of 22 types of immune cells; (B) The heat map of 22 types of immune cells; (C) The difference of immune infiltration between IPAH and normal controls. The normal control group was marked as blue color and IPAH group was marked as red color. P-values < 0.05 were considered as statistically significant; (D) Correlation heatmap of the 22 types of immune cells. Blue presents a negative correlation, red represents a positive correlation, the darker the color, the stronger the correlation.

as far as we known, this is the first time potential biomarkers related to immune infiltration in IPAH have been identified.

Animal models and human studies have revealed that inflammatory mediators are upregulated, including chemokines and chemokine receptors in $\operatorname{IPAH~}(10,11)$, such as CXCL10/CXCR3, CXCL8/CXCR1/CXCR2, CCL2/CCR2, CXCL12/CXCR4/ACKR3, CX3CL1 /CX3CR1, CCL5/CCR5/CCR1 (10, 33). The circulating cytokines were also increased in PAH, including IL-8, IL-10, IL-1 $\beta$, IL-2, IL-4, IL-6, IL-12p70, IL-18, and TNF $\alpha$ (34). Similar to previous findings, the DEGs showed an enrichment in chemokine binding, chemokine receptor activity in this study. Other than their common role in the recruitment of immune cells in response to inflammation, chemokines are also involved in cytokine expression, pulmonary artery cell proliferation, and migration, and. Additionally, chemokines also participate in PAH progression $(35,36)$. And IL-10, IL-6, IL-8, and IL-12p70 have been found to predict the survival of PAH patients (34). Given the evidence that several chemokines and chemokine receptors are increased in $\mathrm{PAH}$, some studies have specifically investigated pharmacological agents targeting chemokines and their receptors in different types of $\mathrm{PAH}$; however, none of these agents have moved to clinical trials despite some inspiring findings.

Inflammatory cell infiltration was also observed around remodeled arteries in animal models (12) and human PAH, such as the formation of a tertiary lymphoid follicle consisting of $\mathrm{T}$ and $\mathrm{B}$ lymphocytes, and DCs have been found near remodeled arteries in IPAH, and DCs occur in the adventitia and media of muscular pulmonary arteries in human IPAH (13). A previous study observed significantly fewer CD8+ T cells and more $\mathrm{T}$ regulatory cells were found in the peripheral blood of patients with IPAH compared with the controls (14). In addition, mast cells were elevated in the pulmonary arteries of IPAH compared with control subjects and may participate in vascular remodeling (15). IgG antibodies from patients with IPAH expressed obvious reactivity profiles with macrovascular and microvascular endothelial cell (37) and fibroblast antigens (38). However, we showed that patients with IPAH had a higher level of CD8 $+\mathrm{T}$ cells, resting memory CD4 $+\mathrm{T}$ cells, gamma delta $\mathrm{T}$ cells, $\mathrm{M} 1$ macrophages, resting mast cells, and a lower level of naïve CD4+ T cells, monocytes, M0 macrophages, activated mast cells, and neutrophils compared with the control group. This discrepancy may be explained 


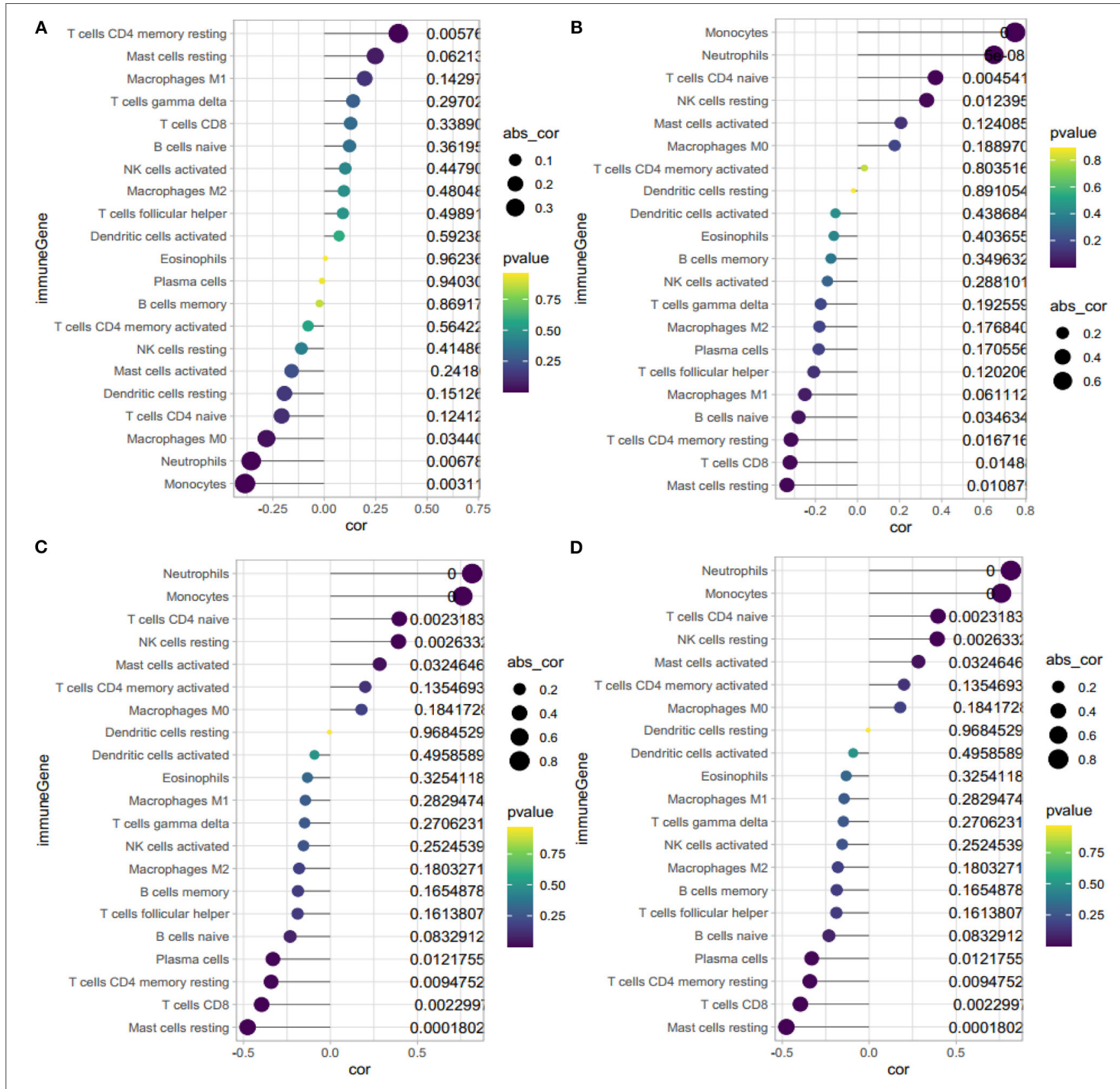

FIGURE 8 | Correlation between HBB, RNASE2, S100A9, IL1R2 and immune cells. (A) Correlation between HBB and immune cells. (B) Correlation between RNASE2 and immune cells. (C) Correlation between S100A9 and immune cells. (D) Correlation between IL1R2 and immune cells. The size of the dots represents the strength of correlation between gene biomarkers and immune cells. The color of the dots represents the $P$-value.

by the fact that CIBERSORT algorithm tends to systematically over- or under-estimate cell types despite a considerably lower estimation bias. As inflammation is involved in many aspects of IPAH, it is plausible to introduce anti-inflammatory drugs into the treatment plan for IPAH, however, studies attempted to find effective anti-inflammatory agents are lacking in IPAH (39).

Four prognostic biomarkers were established according to SVM-RFE and LASSO regression. $H B B$ encodes the HBB protein, which is a key component of hemoglobin, a molecule important for oxygen delivery to tissues. Cell-free hemoglobin was increased in patients with PAH compared with healthy individuals, and positively correlated with $\mathrm{mPAH}$ and PVR and inversely with cardiac index in patients with PAH (40). Proteins encoded by S100A9 and S100A8 constitute calprotectin, a heterodimeric calcium-binding protein. They can be expressed by a broad range of cell types, such as neutrophils, monocytes, early differentiation states of macrophages, and keratinocytes. 
Calprotectin can induce inflammation by binding to cell surface receptors. Under normal conditions, the concentrations are very low, whereas increased obviously in inflammatory diseases (41). Calprotectin also induces apoptosis in tumors at high extracellular concentrations, whereas they facilitate proliferation of tumor cells at lower levels. Cells exposing to high concentration of S100A8/S100A9 caused caspase activation (42). In our study, S100A9 and S100A8 (Figure 6A) were both downregulated in IPAH compared with the control group. It is acknowledged that a cancer-like increase in cell proliferation and resistance to apoptosis is one of the characteristics of IPAH. So we speculated that the lower level of S100A9 and $S 100 A 8$ may contribute to anti-apoptosis in IPAH, and S100A8/S100A9 may be a potential treatment in IPAH.Rnase2 encoded by RNASE2, is a cytotoxic protein secreted mainly by eosinophils as well as macrophages. Rnase 2 has antiviral activity and chemotactic activities in vitro $(43,44)$. Yang et al. revealed that Rnase 2 can activate human DCs resulting in the production of several inflammatory cytokines, chemokines, growth factors, and soluble receptors. RNASE2 also induced maturation of DCs (45). In this study, we found that RNASE2 was downregulated in IPAH, and it had a positive correlation with neutrophils $(r=0.65)$ and monocytes $(r=0.75)$, we suggested that RNASE2 in IPAH was a reflection of immune dysfunction. However, the Rnase2 level in lung tissue should be further validated by western blot. IL1R2 is a member of the interleukin-1 receptor family, which negatively regulate the IL1 system. The IL-1 system is associated with host responses to infections, inflammation, and activation of lymphoid cells $(46,47)$. IL1R2 expression is positively regulated by several anti-inflammatory mediators, in contrast, pro-inflammatory molecules and chemokines repress $I L-1 R 2$ expression (48). In our study, IL1R2 was significantly downregulated in IPAH compared with the control group. We suggested that the inflammation and the high level of chemokines contributed to the reduced level of IL1R2. In brief, these four feature genes are associated with oxygen delivery, immune dysfunction,

\section{REFERENCES}

1. Nazzareno G, Marc H, Jean-Luc V, Simon G, Irene L, Adam T, et al. 2015 ESC/ERS guidelines for the diagnosis and treatment of pulmonary hypertension. Rev Esp Cardiol. (2016) 69:177. doi: 10.1016/j.rec.2016.01.002

2. Kanwar M, Raina A, Passineau M, Benza R. Idiopathic pulmonary arterial hypertension: evolving therapeutic strategies. Semin Respir Crit Care Med. (2017) 38:606-8. doi: 10.1055/s-0037-1606317

3. Cao JY, Wales KM, Cordina R, Lau EMT, Celermajer DS. Pulmonary vasodilator therapies are of no benefit in pulmonary hypertension due to left heart disease: a meta-analysis. Int J Cardiol. (2018) 273:21320. doi: 10.1016/j.ijcard.2018.09.043

4. Spiekerkoetter E, Kawut SM, de Jesus Perez VA. New and emerging therapies for pulmonary arterial hypertension. Annu Rev Med. (2019) 70:4559. doi: 10.1146/annurev-med-041717-085955

5. Aldred MA, Vijayakrishnan J, James V, Soubrier F, Gomez-Sanchez MA, Martensson G, et al. BMPR2 gene rearrangements account for a significant proportion of mutations in familial and idiopathic pulmonary arterial hypertension. Hum Mutat. (2006) 27:212-3. doi: 10.1002/humu.9398 inflammation, and may be involved in the process of antiapoptosis in IPAH.

However, this study has its own limitations. First, the conclusions drawn from bioinformatics analysis require RT-PCR in clinical samples to further substantiate claims. Second, there were unavoidable limitations of CIBERSORT. For example, it fails to provide $P$-values for the detection limits of individual cell types. Moreover, CIBERSORT tends to overesterestimate or underestimate some cell types systematically in spite of a considerably lower estimation bias than other methods (30).

\section{CONCLUSION}

$H B B, R N A S E 2, S 100 A 9$, and IL1R2 were potential diagnostic genes in IPAH. Inflammation and immune dysfunction involves in the pathogenesis of IPAH, pharmacological agents targeting chemokines and their receptors, and anti-inflammatory drugs may be potential effective treatments in IPAH in the future.

\section{DATA AVAILABILITY STATEMENT}

Publicly available datasets were analyzed in this study. This data can be found here: https://www.ncbi.nlm.nih.gov/geo/; GSE117261, GSE48149.

\section{AUTHOR CONTRIBUTIONS}

$\mathrm{HZ}$ is the principle investigator and performed data management and bioinformatics analysis. $\mathrm{HZ}$ and XL conducted statistical analysis and draft the manuscript. $\mathrm{HZ}, \mathrm{XL}$, and $\mathrm{YZ}$ edited and revised the manuscript. All authors contributed to the article and approved the submitted version.

\section{ACKNOWLEDGMENTS}

The authors acknowledge the Gene Expression Omnibus (GEO) database for providing data of IPAH available for free.

6. Shintani M, Yagi H, Nakayama T, Saji T, Matsuoka R. A new nonsense mutation of SMAD8 associated with pulmonary arterial hypertension. J Med Genet. (2009) 46:331-7. doi: 10.1136/jmg.2008. 062703

7. Harrison RE, Berger R, Haworth SG, Tulloh R, Mache CJ, Morrell NW, et al. Transforming growth factor- $\beta$ receptor mutations and pulmonary arterial hypertension in childhood. Circulation. (2005) 111:435-41. doi: 10.1161/01.CIR.0000153798.78540.87

8. Chida A, Shintani M, Nakayama T, Furutani Y, Hayama E, Inai K, et al. Missense mutations of the BMPR1B (ALK6) gene in childhood idiopathic pulmonary arterial hypertension. Circ J. (2012) 76:15018. doi: 10.1253/circj.CJ-11-1281

9. McLaughlin VV, Hoeper MM, Channick RN, Chin KM, Delcroix M, Gaine $\mathrm{S}$, et al. Pulmonary arterial hypertension-related morbidity is prognostic for mortality. J Am Coll Cardiol. (2018) 71:752-63. doi: 10.1016/j.jacc.2017. 12.010

10. Price LC, Wort SJ, Perros F, Dorfmüller P, Huertas A, Montani D, et al. Inflammation in pulmonary arterial hypertension. Chest. (2012) 141:21021. doi: $10.1378 /$ chest.11-0793 
11. Madjdpour C, Jewell UR, Kneller S, Ziegler U, Schwendener R, Booy C, et al. Decreased alveolar oxygen induces lung inflammation. Am J Physiol Lung Cell Mol Physiol. (2003) 284:L360-7. doi: 10.1152/ajplung.00158.2002

12. Hong KH, Lee YJ, Lee E, Park SO, Han C, Beppu H, et al. Genetic ablation of the Bmpr2 gene in pulmonary endothelium is sufficient to predispose to pulmonary arterial hypertension. Circulation. (2008) 118:72230. doi: 10.1161/CIRCULATIONAHA.107.736801

13. Perros F, Dorfmüller P, Souza R, Durand-Gasselin I, Mussot S, Mazmanian M, et al. Dendritic cell recruitment in lesions of human and experimental pulmonary hypertension. Eur Respir J. (2007) 29:462-8. doi: 10.1183/09031936.00094706

14. Ulrich S, Nicolls MR, Taraseviciene L, Speich R, Voelkel N. Increased regulatory and decreased CD8+ cytotoxic T cells in the blood of patients with idiopathic pulmonary arterial hypertension. Respiration. (2008) 75:27280. doi: 10.1159/000111548

15. Montani David, Perros Frédéric, Gambaryan Natalia, Girerd Barbara, Dorfmuller Peter, Price Laura C, et al. C-kit-positive cells accumulate in remodeled vessels of idiopathic pulmonary arterial hypertension. Am J Respir Crit Care Med. (2011) 184:116-23. doi: 10.1164/rccm.201006-0905OC

16. Zhao EF, Xie H, Zhang YS. Identification of differentially expressed genes associated with idiopathic pulmonary arterial hypertension by integrated bioinformatics approaches. J Comput Biol. (2020) 28:7988. doi: $10.1089 / \mathrm{cmb} .2019 .0433$

17. Zhao EF, Xie H, Zhang YS. Predicting diagnostic gene biomarkers associated with immune infiltration in patients with acute myocardial infarction. Front Cardiovasc Med. (2020) 7:586871. doi: 10.3389/fcvm.2020.586871

18. Ritchie ME, Phipson B, Wu D, Hu YF, Law CY, Shi W, et al. limma powers differential expression analyses for RNA-sequencing and microarray studies. Nucleic Acids Res. (2015) 43:e47. doi: 10.1093/nar/gkv007

19. Yu GC, Wang LG, Han YY, He QY. ClusterProfiler: an R package for comparing biological themes among gene clusters. OMICS. (2012) 16:28487. doi: $10.1089 /$ omi.2011.0118

20. Cedric G. ggplot2: elegant graphics for data analysis. J R Stat Soc Ser A Stat Soc. (2011) 174:678-9. doi: 10.1111/j.1541-0420.2011.01616.x

21. Luo WJ, Brouwer C. Pathview: an R/Bioconductor package for pathwaybased data integration and visualization. Bioinformatics. (2013) 29:18301. doi: 10.1093/bioinformatics/btt285

22. Subramanian A, Tamayo P, Mootha VK, Mukherjee S, Ebert BL, Gillette MA, et al. Gene set enrichment analysis: a knowledge-based approach for interpreting genome-wide expression profiles. Proc Natl Acad Sci USA. (2005) 102:15545-50. doi: 10.1073/pnas.0506580102

23. Heikamp K, Bajorath J. Support vector machines for drug discovery. Expert Opin Drug Discov. (2014) 9:93-104. doi: 10.1517/17460441.2014.866943

24. Guyon I, Weston J, Barnhill S, Vapnik V. Gene selection for cancer classification using support vector machines. Mach Learn. (2002) 46:389422. doi: 10.1023/A:1012487302797

25. Tibshirani R. Regression shrinkage and selection via the lasso. J R Stat Soc Ser B Stat Methodol. (1996) 73:273-82. doi: 10.1111/j.1467-9868.2011.00771.x

26. Suykens J, Vandewalle J. Least squares support vector machine classifiers. Neural Process Lett. (1999) 9:293-300. doi: 10.1023/A:1018628609742

27. Friedman J, Hastie T, Tibshirani R. Regularization paths for generalized linear models via coordinate descent. J Stat Softw. (2010) 33:1-22. doi: 10.18637/jss.v033.i01

28. Huang ML, Hung YH, Lee WM, Li RK,Jiang BR, Ding S. SVM-RFE based feature selection and taguchi parameters optimization for multiclass SVM classifier. Sci World J. (2014) 2014:795624. doi: 10.1155/2014/795624

29. Dailey AL. Metabolomic bioinformatic analysis. Methods Mol Biol. (2017) 1606:341-52. doi: 10.1007/978-1-4939-6990-6_22

30. Newman AM, Liu CL, Green MR, Gentles AJ, Feng WG, Xu Y, et al. Robust enumeration of cell subsets from tissue expression profiles. Nat Methods. (2015) 12:453-7. doi: 10.1038/nmeth.3337

31. Hu KJ. Become competent within one day in generating boxplots and violin plots for a novice without prior R experience. Methods Protoc. (2020) 3:64. doi: $10.3390 / \mathrm{mps} 3040064$
32. Friendly M. Corrgrams: exploratory displays for correlation matrices. Am Stat. (2002) 56:316-24. doi: 10.1198/000313002533

33. Mamazhakypov A, Viswanathan G, Lawrie A, Schermuly RT, Rajagopal S. The role of chemokines and chemokine receptors in pulmonary arterial hypertension. Br J Pharmacol. (2021) 178 :72-89. doi: 10.1111/bph.14826

34. Jafri S, Ormiston ML. Immune regulation of systemic hypertension, pulmonary arterial hypertension, and preeclampsia: shared disease mechanisms and translational opportunities. Am J Physiol Regul Integr Comp Physiol. (2017) 313:R693-705. doi: 10.1152/ajpregu.00259.2017

35. Morrell NW, Aldred MA, Chung WK, Elliott CG, Nichols WC, Soubrier F, et al. Genetics and genomics of pulmonary arterial hypertension. Eur Respir J. (2019) 53:1801899. doi: 10.1183/13993003.01899-2018

36. Evans JD, Girerd B, Montani D, Wang XJ, Galiè N, Austin ED, et al. BMPR2 mutations and survival in pulmonary arterial hypertension: an individual participant data meta-analysis. Lancet Respir Med. (2016) 4:129-37. doi: 10.1016/S2213-2600(15) 00544-5

37. Tamby MC, Chanseaud Y, Humbert M, Fermanian J, Guilpain P, Garcia-dela-Peña-Lefebvre $P$, et al. Anti-endothelial cell antibodies in idiopathic and systemic sclerosis associated pulmonary arterial hypertension. Thorax. (2005) 60:765-72. doi: 10.1136/thx.2004.029082

38. Tamby MC, Humbert M, Guilpain P, Servettaz A, Dupin N, Christner JJ, et al. Antibodies to fibroblasts in idiopathic and scleroderma-associated pulmonary hypertension. Eur Respir J. (2006) 28:799-807. doi: 10.1183/09031936.06.00152705

39. Voelkel NF, Tamosiuniene R, Nicolls MR. Challenges and opportunities in treating inflammation associated with pulmonary hypertension. Expert Rev Cardiovasc Ther. (2016) 14:939-51. doi: 10.1080/14779072.2016.1180976

40. Brittain EL, Janz DR, Austin ED, Bastarache JA, Wheeler LA, Ware LB, et al. Elevation of plasma cell-free hemoglobin in pulmonary arterial Hypertension. Chest. (2014) 146:1478-85. doi: 10.1378/chest.14-0809

41. Shabani F, Farasat A, Mahdavi M, Gheibi N. Calprotectin (S100A8/S100A9) : a key protein between inflammation and cancer. Inflamm Res. (2018) 67:801-12. doi: 10.1007/s00011-018-1173-4

42. Srikrishna G. S100A8 and S100A9: new insights into their roles in malignancy. J Innate Immun. (2012) 4:31-40. doi: 10.1159/000330095

43. Rosenberg HF. Eosinophil-derived neurotoxin (EDN/RNase 2) and the mouse eosinophil-associated RNases (mEars): expanding roles in promoting host defense. Int J Mol Sci. (2015) 16:15442-55. doi: 10.3390/ijms160715442

44. Koczera P, Martin L, Marx G, Schuerholz T. The ribonuclease A superfamily in humans: canonical RNases as the buttress of innate immunity. Int J Mol Sci. (2016) 17:1278. doi: 10.3390/ijms17081278

45. Yang D, Chen Q, Rosenberg HF, Rybak SM, Newton DL, Wang ZY, et al. Human ribonuclease A superfamily members, eosinophil-derived neurotoxin and pancreatic ribonuclease, induce dendritic cell maturation and activation. J Immunol. (2004) 173:6134-42. doi: 10.4049/jimmunol.173. 10.6134

46. Dinarello CA. Anti-inflammatory agents: present and future. Cell. (2010) 140:935-50. doi: 10.1016/j.cell.2010.02.043

47. Garlanda C, Dinarello CA, Mantovani A. The interleukin-1 family: back to the future. Immunity. (2013) 39:1003-18. doi: 10.1016/j.immuni.2013.11.010

48. Molgora M, Supino D, Mantovani A, Garlanda C. Tuning inflammation and immunity by the negative regulators IL-1R2 and IL-1R8. Immunol Rev. (2018) 281:233-47. doi: 10.1111/imr.12609

Conflict of Interest: The authors declare that the research was conducted in the absence of any commercial or financial relationships that could be construed as a potential conflict of interest.

Copyright (c) 2021 Zeng, Liu and Zhang. This is an open-access article distributed under the terms of the Creative Commons Attribution License (CC BY). The use, distribution or reproduction in other forums is permitted, provided the original author(s) and the copyright owner(s) are credited and that the original publication in this journal is cited, in accordance with accepted academic practice. No use, distribution or reproduction is permitted which does not comply with these terms. 